\title{
Impossible généalogie littéraire de René Maran
}

\section{Ferroudja Allouache}

URL : https://journals.openedition.org/coma/7064

DOI : 10.4000/coma.7064

ISSN : 2275-1742

\section{Éditeur}

Institut des textes \& manuscrits modernes (ITEM)

\section{Référence électronique}

Ferroudja Allouache, «Impossible généalogie littéraire de René Maran », Continents manuscrits [En ligne], 17 | 2021, mis en ligne le 15 octobre 2021, consulté le 07 janvier 2022. URL : http:// journals.openedition.org/coma/7064 ; DOI : https://doi.org/10.4000/coma.7064

Ce document a été généré automatiquement le 7 janvier 2022.

\section{(c) (i) ()}

Continents manuscrits - Génétique des textes littéraires - Afrique, Caraîbe, dispora est mis à disposition selon les termes de la licence Creative Commons Attribution - Pas d'Utilisation Commerciale - Pas de Modification 4.0 International. 


\title{
Impossible généalogie littéraire de René Maran
}

\author{
Ferroudja Allouache
}

\section{Introduction}

1 Impossible généalogie : pourquoi ce titre ? D’abord parce que René Maran était l'un des grands absents des manuels du secondaire sur lesquels je m'appuyais pour faire découvrir la littérature française et francophone aux élèves. Le poète, romancier, essayiste, chroniqueur littéraire guyanais n'était ni avec ses pairs français ou européens ni avec les écrivains dits francophones, souvent classés en fin des anthologies, quand celles-ci leur accordaient une place. Il était absent. Comme suspendu dans le vide. Sans histoire. Sans lien.

2 Pourtant, contrairement aux intellectuels afro-antillais ou maghrébins qui suivront, ceux qui serviront de jalons aux anthologues francophones pour dater la naissance de la littérature négro-africaine, maghrébine d'expression française et d'autres syntagmes qui n'en finiront pas de dire la difficulté - voire l'impossibilité - de nommer les textes des écrivains anciennement sous domination, sujets de l'Empire, Maran n'a pas revendiqué, de son temps, une quelconque appartenance autre que celle qui le lie indéfectiblement à la France, « pays d'élection ».

3 Un siècle après la parution de Batouala, il m'a semblé pertinent de revisiter deux temporalités distinctes afin de comprendre les mécanismes de filiation/affiliation, de généalogie littéraire dans quelques organes de presse de l'époque et anthologies de littératures parues dès les années 1960. En somme, il s'agit de revisiter la mémoire de la littérature, d'interroger les discours relayés par la presse afin de redécouvrir celle du temps de Maran, moment de la réception et des réactions immédiates des chroniqueurs qui sont en train de "faire la littérature", de légitimer ou non une œuvre et de questionner celle, contemporaine, qui rend compte du vieillissement des textes, de l'invention sans cesse renouvelée, de leur insertion dans le champ littéraire. 
Ce sont les secrets de fabrication de la ressemblance ou de distinction/différenciation radicale à l'œuvre dans les discours des chroniqueurs littéraires et dans les anthologies qui m'intéressent car ils éclairent l'ancrage dans la mémoire littéraire. Intertextualité, ressemblance, mémoire, ces concepts ont une importance fondamentale dans l'élaboration de l'histoire littéraire francophone à l'intérieur de la République mondiale des lettres. Maran est exemplaire par la façon dont il est doublement mis à l'écart, à côté, comme s'il n'était ni de son temps ni dans son temps, jamais à sa place dans l'histoire française/ francophone/ mondiale.

5 Onze journaux ont été recensés afin de saisir l'actualité de l'époque, au moment où paraît Batouala : ils vont de la veille de l'attribution du prix (avant le 15 décembre) 1921 à l'année 1922. Ils sont de tendance monarchiste-conservatrice (L'Action française, Le Petit Journal, Le Gaulois), chrétienne catholique (La Croix), conservatrice (Le Temps, L'Écho de Paris), de droite (Le Figaro) ou de gauche (Le Petit Parisien, L'Humanité, L'Écho d'Alger). Le compte rendu de la Nouvelle Revue française, paru en janvier 1922, apporte un éclairage intéressant puisqu'il s'agit d'une critique centrée sur l'esthétique et, qui plus est, publié dans une prestigieuse revue qui se veut avant tout apolitique.

\section{Écrire la mémoire du présent : la résistance face à la ressemblance}

6 L'on retient du prix Goncourt 1921 le scandale qu'a suscité Batouala, surtout la préface dans laquelle Maran s'en prend aux abus d'une frange de l'administration coloniale. Or, une lecture attentive de la presse permet de mieux saisir l'impensé qui travaille consciemment ou non les positions des journalistes, des médias de l'époque et les résistances des milieux littéraires parisiens à aborder l'œuvre sans préjugés raciaux. L'écrivain est sans cesse montré du doigt comme «nouveau » venu à la Civilisation, celle qui lui a tendu la main, l'a arraché à son milieu, à sa culture, à sa langue; il doit rester au seuil. Seuil impossible à franchir : seuil de la langue, seuil de la littérature (ici le roman colonial, devenu un sous-genre autonome), seuil de la Nation. La lecture/ réception de l'œuvre procède souvent sur le mode du rejet, qu'il soit d'ordre linguistique, intertextuel ou moral. Tout se passe comme si l'idée pernicieuse mais souterraine qui irrigue les discours critiques était la difficulté, pour les chroniqueurs, à se reconnaître, à se retrouver dans le récit de Maran, bien que celui-ci évoque un contexte connu, réfère à une réalité proche - certes décalée - des textes littéraires sur l'Afrique.

\section{L'élément linguistique}

7 La langue est un élément clé de la mémoire du présent : elle fait la littérature. C'est par la langue que les textes lus, traduits, commentés sont ensuite insérés dans le corpus toujours plus étendu de ce qui constitue et enrichit en permanence le patrimoine. La langue-littérature est étroitement liée à l'idée de l'identité-Nation.

8 C'est sur ce point qu'il nous semble important d'attirer l'attention: la langue est un instrument politique surtout quand il est question des écrivains dits francophones. Pour Maran, cette question n'aurait pas dû et ne devrait pas se poser puisqu'il est né « dedans ». Le talent s'exprime avant tout par le "génie » d'une langue, ici le français. Or, Maran est très vite attaqué sur son français, son style. Les trouvailles, les inventions 
sont suspectes, mal reçues, incomprises. Benjamin Crémieux, qui salue en Batouala « un grand souci d'exotisme", en souligne néanmoins les manques: l'absence d'un lexique en fin d'ouvrage utile pour une meilleure compréhension ${ }^{1}$. Le motif de la déception donne l'impression d'une attente déjà programmée et à laquelle l'auteur fait défaut, comme si la réception ne devait produire aucune surprise pour le chroniqueur interprète et intermédiaire entre l'œuvre et le public restreint de la prestigieuse Nouvelle Revue française. La déception traduirait implicitement la difficulté à saisir l'originalité d'un récit en rupture avec les textes appartenant au genre du « roman colonial ", dont le souci exotique consiste à faire parler "petit nègre " les indigènes, stéréotypes auxquels ont été habitués de longue date les lecteurs. Cependant, poursuit Crémieux, Batouala «se limite presque uniquement à l'emploi de vocables nègres ». Les deux adverbes «presque uniquement » renchérissent l'idée de cette attente qui n'est pas là, de l'inattendu, d'un manque. Il va même jusqu'à corriger, reformuler certaines phrases données en exemple et qui témoignent de l'étrangeté que produit la lecture du texte :

«les sons discordants des balafons et des koundés s'unissaient au tam-tam des li'nghas» peut suggérer une musique barbare. Mais un cliquetis de syllabes incompréhensibles, s'il peut suggérer quelques grossières images auditives, ne pourra en aucun cas suggérer d'images visuelles. «Ils avaient quitté leur kagas, leur brousse, leur patas-patas boueux» n'en dit pas plus en vérité que «Ils avaient quitté leur brousse $»^{2}$.

Quant au style de Maran, sa langue, la question rhétorique laisse dubitatif le lecteur: «Veut-on maintenant une idée du français tel que l'écrit M. Maran? ». Il cite pêle-mêle des fragments de la préface et des extraits du roman sans aucune distinction. Aucun commentaire n'accompagne son choix. Le lecteur est abandonné à lui-même pour en saisir le sens.

La langue est aussi un argument dont usent les journalistes pour épingler Maran de façon plus virulente et violente. Le chroniqueur de La Croix interroge le statut d'auteur de Maran dont il juge qu'il "vient de naître à la littérature française " et récuse l'authenticité de l'œuvre pleine de «fautes nombreuses que son inexpérience excuse ${ }^{3}$ ». Maran n'aurait pas de talent. Il est mis à l'index, accusé d'être un nouveau venu, qui plus est inexpérimenté. Lui est reproché le style «tarabiscoté et sans charme » mis au compte, encore une fois, de sa méconnaissance du français, manière euphémisée de rappeler sans aucun doute une généalogie éloignée de celle de ses pairs français : «il ne dépasse pas le travail d'un écolier ", note ironiquement Maurice de Waleffe, homme de lettres et journaliste belge dans $L^{\prime} E c h o d^{\prime}$ Alger $^{4}$, organe de presse qui orchestre l'«éreintement littéraire» du roman. Dans le même journal, F. Ancey attaque ouvertement Maran pour avoir touché à la belle langue, à sa grammaire, la pureté du symbole du " génie français » : " ce livre n'est même pas écrit en français ", affirme-t-il. Selon lui, Maran manque d'égard pour «l'esprit élevé de notre grammaire ${ }^{5}$ ». Le possessif marque une distance, une frontière entre un nous incarné par la langue, et l'Autre-étranger, extérieur à l'imaginaire que convoque Ancey. Maran est expulsé de cet imaginaire comme si implicitement lui était défendu l'appropriation de cet idiome. Il ne peut lui appartenir comme il l'est «naturellement» pour «nous». Ancey n'apprécie pas les mots nouveaux tels que «bombillement, vocération, copuler, chicotter ", relève des phrases « qui dénotent une connaissance très rudimentaire de la langue française » telles que «Les cabris sollicitent leurs "femelles" ", «Le jour tôt 
survient où l'on est malpropre, même à copuler ", "l'ivresse annule jusqu'au regret possible avec la conscience ${ }^{6} »$.

11 Le relevé glisse insidieusement vers un choix éloquent de citations extraites du roman pour en discréditer la valeur littéraire et, dans le même temps, s'en servir pour exposer les réels dangers qu'encourent les Blancs face à la violence, au désir de vengeance des Noirs: «Nous les massacrerons, un jour, très tard». Et de conclure: «Et c'est inévitablement ce qui arrivera, à moins qu'on ne leur ait auparavant donné le droit de citoyen français, l'amour du travail et la possibilité de chasser pacifiquement ces blancs qu'ils rêvent pour l'instant de massacrer ${ }^{7}$."

Que disent en creux ces exemples? Maran n'est-il pas renvoyé à son étrangeté, son extranéité à la langue-Nation? Tout le travail sur la langue: recherche lexicale, tournure syntaxique, intrusion de termes étrangers dans la phrase, en somme ce qui constitue le style d'un auteur, l'originalité du récit, ce qui en fait sa littérarité sont remis en cause. La fiction romanesque déborde sur la réalité, mieux la réalité est rattrapée par la fiction. C'est sur la langue que les critiques se focalisent afin d'entretenir autant que possible l'impossible greffe de ce texte avec d'autres qui participent à la fabrique d'une fiction littéraire nationale, à la création de nouvelles références, d'échos transtextuels. Cette intertextualité peine à émerger dans les commentaires des chroniqueurs.

\section{L'intertextualité, fabrique d'une radicale étrangeté}

13 Le titre peut sembler paradoxal et il l'est puisque, dans le domaine de la littérature, le commentaire a pour objectif de tisser des échos d'un texte à l'autre, d'en exhiber les tissages, reprises, distanciation, d'inscrire un texte dans l'histoire, d'en assurer une lecture, une mise en perspective avec le passé, un lien avec le présent ou une projection dans l'à-venir. Or, la fiction de Batouala se heurte à cette inscription dans l'espacetemps littéraire français, résistance perceptible dans l'économie discursive des chroniqueurs. Si les comptes rendus révèlent un travail de mise en relation du roman avec d'autres récits exotiques dont les thèmes sont similaires, le tissage intertextuel est soumis à un rude travail de sape. Les comparaisons établies fonctionnent la plupart du temps sur une perception dépréciative, une dévaluation systématique du récit ou de l'auteur.

14 Maran est tour à tour comparé à Louis Bertrand, aux frères Tharaud, à Lucie Cousturier, à Pierre Loti, voire Zola et Chateaubriand non pour les résonances thématiques que suscitent les textes entre eux, les dialogues possibles de l'un à l'autre, mais pour en souligner les écarts, une différence qui ne saurait être discutable, parce que la discuter, c'est déjà lui reconnaître un statut dans l'espace généreux que suppose la littérature. L'on rappelle à l'envi que Maran n'aurait pas leur talent, leur génie, point de vue résumé dans l'invective de F. Ancey de L'Écho d'Alger: " histoire banale d'un nègre, de mœurs nègres, écrite par un nègre qui a la haine de la civilisation européenne ${ }^{8}$ ». Ce qui n'est pas dit, ou qui est suggéré en pointillé et qu'il faut entendre, c'est le trouble que provoque la lecture de Batouala: Ancey semble déstabilisé par la préface et le roman, ne pas se retrouver dans le texte qui lui parle autrement de l'exotisme, du Nègre. Or, c'est parce qu'il ne se retrouve pas, qu'il ne s'y reconnait pas que le journaliste va combler le manque, l'absence par le jugement dépréciatif, frisant souvent la condescendance, l'accusation. L'allusion à «la haine du blanc» a une répercussion sur l'actualité 
politique de l'époque : les dissensions franco-allemandes sur la gestion des colonies et la peur de la contestation qui pourrait se manifester orientent volontairement le sens $\mathrm{du}$ roman vers une lecture politique (Batouala est traduit en allemand dès le début de l'année 1922, «ce qui favorisera l'appropriation du texte dans le pays voisin à des fins polémiques » et politiques, rappelle L. Rubiales ${ }^{9}$ ).

Il s'agit donc de maintenir la distance, de montrer l'absence de ressemblance entre Maran/Batouala et le monde de la littérature. Les rappels ne manquent pas, parmi lesquels celui de L'Écho de Paris ( 15 déc. $^{10}$ ) qui affirme que Batouala "n'est pas une peinture colorée comme dans Loti ». Colorer, c'est transformer, donner des couleurs, embellir ce qui est appréhendé (lieux, personnages, mœurs...). En somme, la « doctrine de la couleur locale » dont rend parfaitement compte J. Croisières :

Notez comme un Louis Bertrand, pourtant consciencieux réaliste, colore adroitement sa palette et détermine les éléments constitutifs d'un paysage africain où se trouvent non pas uniquement des corps affublés d'oripeaux, mais encore des âmes éprises de l'idéal chatoyant qui brille au creux du ciel. M. Maran, si je l'ai bien compris, ne doit lire qu'avec effroi les livres de M. Bertrand ${ }^{11}$.

La comparaison entre le réalisme poétique de Bertrand, l'un des théoriciens principaux du roman colonial de son temps, et celui trivial de Maran, passe par l'éloge vs dépréciation qui tend à gommer toute tentative de ressemblance entre pairs. Or, Maran, féru de littérature, n'est pas dupe de ce qui s'écrit. Toute la préface auctoriale résonne/raisonne d'allusions aux textes de philosophes, historiens, gens de lettres et de politique français et étrangers, figures tutélaires dont il se revendique ou discute les idées. Maran dit ce que devrait être un « véritable roman nègre »:

[...] j'ai mis six ans à le parfaire. J'ai mis six ans à y traduire ce que j'avais, là-bas, entendu, à y décrire ce que j'ai vu. [...] pas un moment je n'ai cédé à la tentation de dire mon mot. J'ai poussé la conscience objective jusqu'à y supprimer des réflexions qu'on aurait pu m'attribuer. [...] Il faut que vous [ses frères en esprit] aidiez ceux qui disent les choses telles qu'elles sont, non telles qu'on voudrait qu'elles fussent ${ }^{12}$.

L'illusion est brisée : le souhait de dire les choses telles qu'elles sont et non telles qu'on voudrait qu'elles soient place l'auteur dans la tradition du roman réaliste sans pour autant exclure le clin d'œil évident à l'intertexte du récit colonial avec lequel il prend des distances: Batouala n'est pas un récit dont la vocation serait de faire aimer les colonies, comme l'affirmait Delavignette. Maran refuse de tronquer le réel, de l'amputer de ce qui est et qui ne peut plus être dissimulé, idée que souligne L'Humanité qui alterne un choix d'extraits évocateurs de la préface en lien avec d'autres du roman $^{13}$. Pour la première fois, il n'est plus question de « nègres d'idylles à la Bernardin de St-Pierre ", souligne l'organe communiste qui entend se servir de l'œuvre à des fins politiques. Le retentissement avec l'actualité n'échappe à aucun journaliste.

Maran est davantage sommé de se justifier pour avoir peint des «nègres moins aimables que ceux de Mme Lucie Cousturier ", ou moins admiratifs de la culture du Blanc comme avait pu l'être le Samba Diouf des frères Tharaud, s'indigne Chaumeix du Gaulois $^{14}$. Ces derniers comprennent le Nègre parce qu'ils ont su colorer ce qu'ils percevaient en lui. Chaumeix place l'Autre en position subalterne, inférieure : «Ces races Ouolofs et Mandingues, ces Malinkés, Diolas et Toucouleurs, aux beaux noms, apparaissent dans le livre des Tharaud comme nos frères, moins évolués que nous, évidemment, mais en tout cas ce ne sont nullement des monstres. ${ }^{15}$ » La proximité avec l'autre qu'introduit la comparaison chute brusquement dans la dévaluation («moins 
évolués») que renforce l'adverbe "évidemment», rappelant ainsi que tout rapprochement est impossible.

Les discours des journalistes sont émaillés de formules stéréotypées dépréciatives, de jeux de mots fort peu subtils qui invalident la reconnaissance de l'œuvre, son affiliation à l'imaginaire littéraire de son temps : c'est du "goncourisme de la brousse ", s'indigne Paul Souday du Temps (15 déc.), du « romantisme de couleur », écrit G. Bauer de L'Écho de Paris (15 déc.). D'autres cultivent la condescendance comme La Croix qui qualifie le livre de « réalisme africain ${ }^{16}$ ».

Maran s'inscrit dans et contre les modèles établis et reconnus de son temps. Si Batouala dialogue avec la mémoire de l'histoire littéraire hexagonale, avec l'intertexte colonial en particulier, force est de constater qu'il s'en écarte. La narration est prise en charge par le sujet colonisé. L'irruption du Nègre maniant savamment la langue perturbe. Pis encore, il discourt sur ce qu'on lui impose par la violence et, détail d'importance, renseigne le lectorat éloigné/ignorant, sur son histoire, celle de l'avant-venue des Blancs. Ce qui est considéré comme la sauvagerie - inventée par le regard extérieur revêt une autre dimension dans/grâce aux monologues intérieurs de Batouala qui rumine, fulmine, s'indigne et se moque ( «Jadis, avant la venue des Blancs, on vivait heureux »). Ce miroir inversé renverse l'ordre discursif dominant : le Blanc n'a plus le monopole de la parole, il ne produit ni n'entretient d'images d'Épinal, d'imaginaire fantasmé sur l'Autre-Sauvage-Indigène-Nègre qui lui devait son existence. «Il n'y est plus question des blancs mais des noirs et de leurs mœurs ${ }^{17}$ ", note G. Bauer quand Le Figaro relève non sans mépris que "l'on réhabilite ainsi, à bien juste titre, les malheureux nègres qui pullulent dans la littérature " (15 déc.). Le renversement de situation est radical. Ainsi, l'espace discursif devient une mise en scène où sont discutés les valeurs, les progrès des Européens, la guerre qu'ils se livrent mutuellement (Frandjés et Zalémans), le motif véritable de leur venue et les raisons de l'asservissement des populations, à travers la variation des points de vue.

\section{La dimension morale}

L'immoralité est un autre argument que brandissent les chroniqueurs pour discréditer la reconnaissance de l'œuvre par le prestigieux prix Goncourt. Batouala est accueilli comme un récit dépourvu de morale, violent, aux "germes malsains, aux idées haineuses [...] : la trivialité des termes, la grossièreté de certaines descriptions font que ce roman ne peut être lu par des jeunes filles ${ }^{18}$ ». Il est décrit comme "un méchant livre, outrancier et faux » dont il faut "décourager l'exportation ${ }^{19}$ ». En somme, la critique anti-Batouala est unanime sur le rôle qui est assigné à la littérature : éduquer, instruire ; mieux, continuer à entretenir l'imaginaire littéraire au service des progrès de l'Empire colonial. Après tout, les théoriciens de la littérature coloniale tel Delafosse rappelaient que son but est de «faire connaitre et aimer nos colonies ${ }^{20}$ ». Or Batouala fonctionne comme une contre-fiction de l'inconscient collectif. «La littérature est une manifestation de l'existence des réalités de l'histoire ${ }^{21}$. " Le récit dérange, perturbe le discours idéologique dominant et montre combien la littérature est une puissante machine à déconstruire l'Histoire fantasmée des peuples et territoires conquis et à proposer un contre-récit.

22 F. Ancey prévient le lecteur sur ce qu'il juge immoral dans Batouala et qu'il faut condamner : 
Cette fête où hommes, femmes et enfants sont conviés pour assister à la circoncision des mâles et à "l'excision » (!) des femmes, et qui se termine dans des débordements ignobles, n'est qu'une scène de pornographie répugnante qui ne saurait avoir sa place dans un roman honnête et qui doit respecter ses lecteurs et lectrices.

Ce sont des plaies hideuses d'une littérature qu'il faut tenir cachées et non pas étaler au grand jour de la réclame tapageuse d'un prix Goncourt [... ${ }^{22}$.

La connivence avec le public est tacite : elle s'élabore sur la perception tronquée des mœurs locales que soulignent les adjectifs axiologiques "ignobles ", "répugnante ", « hideuses » et que F. Ancey impute à un manque de respect pour les lecteurs/lectrices. Batouala n'a pas «sa place » parmi les romans dignes de ce nom.

C'est aussi de place qu'il est question dans Le Gaulois et Le Temps. La dimension morale $\mathrm{du}$ livre est appréhendée de manière détournée. La critique de $\mathrm{P}$. Souday et A. Chaumeix s'appuie sur une dichotomie permanente pour présenter les personnages indigènes des romanciers français, perçus comme dociles, aimables, éduqués et en constante admiration des contrées métropolitaines - car ils se déplacent de leur village jusqu'à l'Hexagone - et ceux de Maran présentés comme des «nègres débauchés, cruels, perfides, paresseux [...] moins aimables que ceux de Lucie Cousturier ${ }^{23} »$. La romancière est louée pour l'« étude psychologique délicate et idéaliste des tirailleurs sénégalais ", pour sa capacité à déceler en eux " de grands enfants sensibles, pleins de délicatesses morales, gentils et touchants, à la manière de gracieux animaux $»^{24}$. Quant aux frères Tharaud, Chaumeix ne tarit point d'éloges à leur égard :

Le récit de l'arrivée en France des Sénégalais est magnifique. MM. Tharaud nous montre ces nègres, venus de toutes les parties de notre empire colonial, traversant au mois de juin, dans un train encombré, les riches provinces de chez nous. Ils notent leurs réflexions, leurs surprises, leur admiration candide. Ces champs si bien travaillés les émerveillent ; ils ne croyaient pas les blancs si nombreux ni si riches ${ }^{25}$.

Malgré la faveur que rencontre l'art nègre, la réception de Batouala est absorbée par l'actualité politique. Aucune familiarité n'est instituée entre le récit et ceux en vogue à l'époque. Maran reste à la lisière de la littérature, à la marge.

\section{Quelle postérité de Batouala? Anthologies et dictionnaires littéraires}

Que sont devenus Batouala et René Maran quelques décennies plus tard? Que retiennent les anthologies et dictionnaires littéraires de l'essayiste et chroniqueur, du poète et romancier, du fabuliste qui n'a cessé d'écrire tout au long de sa vie? Leur vocation principale est d'offrir un choix de textes, d'auteurs qui constituent la mémoire de la littérature. L'anthologie a pour ambition de proposer une histoire chronologique de la littérature en tentant de rendre compte des idées d'une époque, des tendances, de la créativité des avant-gardes. Elle se donne aussi pour objectif de repenser - parce que l'histoire littéraire est sans cesse renouvelée - les catégories et chronologies, les catégorisations périodiques et d'œuvrer à l'extension, toujours déjà plus importante, du corpus national pour accueillir de nouveaux auteurs européens, étrangers. De fait, l'histoire littéraire établit des liens entre les textes retenus que tout sépare en apparence, fabrique des ressemblances entre les écrits, les auteurs ou établit des frontières. Quelle visibilité anthologies et dictionnaires littéraires français et francophones accordent-ils à l'auteur de Batouala? 
Aucune trace de l'écrivain n'est mentionnée dans le Lagarde et Michard du xxe siècle, dont le titre dit l'exigence du choix opéré: Les Grands Auteurs français. Anthologie et histoire littéraire ${ }^{26}$. Dans la table des matières (p. 892-893), ni la rubrique «Le roman de 1919 à 1939 », ni la sous-rubrique " roman psychologique » n'incluent le nom de Maran aux côtés de Chardonne, Radiguet et d'autres. Il ne figure pas non plus dans la thématique "Critique sociale et peinture des mœurs" auprès de Barbusse, Aragon, Céline, Mac Orlan ${ }^{27}$.

S'il a été reproché à Maran de maltraiter la langue, de ne pas la respecter, le commentaire sur le patois de Ramuz en vaut le détour :

Souvent attaqué pour les particularités de sa langue et de son style, il a lui-même par bravade parlé d'une "espèce de patois natal [...]. Son expression, lorsqu'il écrit ses «vaudoiseries", est donc parfaitement concertée : elle «brise la syntaxe et la grammaire » sans les détruire...28

Les "vaudoiseries" sont reconnues comme un style propre à l'auteur - certes tardivement - et c'est cette reconnaissance singulière qui n'a pas fonctionné pour Maran.

La Littérature française : dynamique et histoire II $^{29}$, ouvrage mené sous la direction de JeanYves Tadié, présente aussi un intérêt pour la partie consacrée à la fin du XIX et début $\mathrm{xx}^{\mathrm{e}}$ siècle qui décrit l'esprit nouveau, le dynamisme de la presse dont de grandes plumes (Proust, A. France...) assurent la « liaison entre la littérature de qualité et le public $^{30}$ ", le rôle de plus en plus important et puissant des revues, dont certaines sont avant-gardistes (Revue des deux mondes, Nouvelle Revue française). Là encore, le nom de Maran ne circule pas dans l'espace dédié aux romans exotiques ou psychologiques. Cependant, à l'exception de Loti, ni L. Bertrand, ni Randau, ni Cousturier et tant d'autres ne sont non plus mentionnés. Enfin, parmi les noms associés aux fictions évoquant les horreurs et ravages psychologiques de la Grande Guerre, reviennent ceux de Paulhan, Giraudoux, Radiguet et Cocteau ${ }^{31}$.

31 René Maran, qui ne s'est jamais considéré autrement qu'auteur français, se retrouve séparé de ses pairs et de son temps. De la période des "années folles ", les noms que retient la postérité sont ceux de Colette, des surréalistes, de Giraudoux... Dans les tableaux chronologiques, à la date de 1921 apparait le nom de Jacques Chardonne, dont La Cavalière Elsa était en concurrence avec Batouala. Il faut noter toutefois que les romans qui avaient pour rôle de mieux faire connaître l'Afrique tels ceux des frères Ary-Leblond, Tharaud, Lucie Cousturier, dont la presse a vanté les mérites, salué le succès contre Batouala, n'ont pas non plus été retenus.

Maran apparaît dans l'anthologie Littérature Textes et Documents $\mathrm{XX}^{e} \mathrm{~s}^{32}$ mais son classement en fin d'ouvrage interpelle. Il est classé en "Littératures francophones ${ }^{33}$ " avec les romanciers d'Afrique noire et des Antilles. Sa constellation littéraire est circonscrite à un espace-temps et une généalogie coupés de l'histoire de la littérature générale, comme si ces intellectuels étaient assignés à une appartenance identitaire et géographique. Avec Damas, Senghor et Césaire, ils sont appréhendés comme les " fondateurs » d'une littérature afro-antillaise francophone. Ainsi, en quelques pages, l'on condense et fabrique une histoire à part, différente, amputée de l'histoire littéraire ${ }^{34}$. Or, un travail sur la bibliothèque de ces intellectuels permettrait de découvrir les références à la tradition littéraire nationale (les troubadours, Rimbaud, Lautréamont...) et universelle (Faulkner, Joyce, Dostoïevski...). La désignation francophone n'est pas définie mais elle dit en creux qu'y sont rassemblées les marges 
auxquelles on suppose une ressemblance culturelle, géographique et historique. C'est la communauté de ceux qui n'ont pas de communauté.

La résistance à mettre en valeur les liens qui unissent ces auteurs à l'espace littéraire français se retrouve dans le Dictionnaire des écrivains de langue française ${ }^{35}$, à l'entrée Maran, même s'il faut saluer l'ambition de l'ouvrage de s'ouvrir aux auteurs francophones (Mammeri, Memmi...). La brève notice résume la vie de Maran à l'Afrique, tente de tricoter des liens entre ses récits animaliers et La Fontaine. À l'inverse, celle de Ramuz, plus détaillée, s'organise en rubriques (narratologie, style, personnages).

L'Encyclopédia Universalis ${ }^{36}$ procède également par classement (auteur, thématique ou aire géographique). Maran est rangé en " Afrique subsaharienne » et sert de jalon pour marquer " une date essentielle »: l'avènement d'une littérature hors de France. Or, le regroupement homogène sous une telle dénomination doit être interrogé. Si les " dictionnaires de littérature servent d'abord à cerner [des] généalogies ${ }^{37}$ ", à retisser les filiations, la catégorisation imposée à Maran et sa généalogie réduite à l'Afrique relèvent-elles d'un impensé que l'on pourrait étendre aux autres auteurs francophones issus des anciennes colonies? Batouala " aurait pu s'inscrire dans la lignée du "roman colonial" ${ }^{38}$ ", est-il écrit, mais la réprobation d'une partie de la presse scandalisée par la préface et le texte a eu raison de l'ingéniosité de Maran.

Qu'en est-il des anthologies francophones? Alors que le romancier et essayiste martiniquais Léonard Sainville saluait en Maran le "pionnier de la littérature antillaise ${ }^{39}$ ", Lilyan Kesteloot contestait, quasiment à la même période, l'appellation : "[u]ne place à part doit être faite à René Maran. Impossible de ranger dans la littérature antillaise cet Antillais qui vécut en France et en Afrique, et qui assimila entièrement et sans effort la culture française $!^{40}$ ». Aussi, Maran a-t-il été tour à tour omis ou à peine évoqué dans de nombreuses anthologies de littérature francophone d'Afrique ou des Antilles. Où le placer : dans la lignée des descendants d'esclaves, dans celle des colonisés? Il semble que l'appréhension de l'écrivain et essayiste est empreinte d'une orientation politique manifeste. Ce que souligne Kesteloot qui avait rencontré Maran en 1959: il est «[c]onsidéré par les noirs comme un précurseur de l'actuelle "négritude", il avoue qu'il la comprend mal et a tendance à y voir un racisme plus qu'une nouvelle forme d'humanisme ${ }^{41}$ ». Elle rappelle que "[r]ejeté par les Français, le roman passa à la postérité négro-africaine ${ }^{42}$ ", et situe l'esthétique de Maran dans la «tradition de Flaubert et de Suarez ». Elle affirme enfin que ses œuvres "n'appartiennent pas à la littérature nègre, quoiqu'elles en aient influencé les idées ${ }^{43}$ ". Kesteloot et Sainville ont tenté de rassembler des auteurs autour de thématiques et de périodisation, même si l'ambition de l'intellectuel martiniquais était d'ouvrir l'anthologie à tous les écrivains noirs du monde. Sa démarche s'inscrit dans la volonté d'une archéologisation des textes et un désir d'historicisation. Bien qu'elle fût originale pour son époque, son entreprise n'a pas été poursuivie, ni repensée dans la réflexion générale sur la littérature afro-antillaise ultérieure.

37 Les anthologies Littérature francophone et Littératures d'Afrique centrale ${ }^{44}$ dirigées par J.L. Joubert fournissent également des éléments de réflexion sur la place de Maran dans la récente appellation du concept francophone/francophonie. Dans Littérature francophone, le découpage par siècles débouche sur un $\mathrm{xx}^{\mathrm{e}} \mathrm{s}$. séparant les auteurs français et les autres ; même le Belge Michaux figure parmi les francophones!

S'ensuivent des pages de synthèse sur chaque aire géographique (Afrique noire, Caraïbe, océan Indien...). Mais tout l'intérêt de cette présentation réside dans le fait que 
tous les auteurs sont regroupés sous des thèmes : les romanciers canadiens côtoient les Sénégalais, les poètes malgaches dialoguent avec ceux de la Caraïbe, et ainsi de suite. Maran inaugure la thématique «La prise de la parole » et est décrit comme «le premier auteur important de la littérature négro-africaine de langue française ${ }^{45}$ ». Curieusement, si Maran est catégorisé écrivain négro-africain, ce qui explique son absence parmi les intellectuels de la Caraïbe, dans le volume dédié aux Littératures d'Afrique centrale, il n'apparaît dans aucun des pays centre-africains mais est classé en fin d'ouvrage, à la rubrique "Rencontres des cultures ", parmi cinq romanciers dont les extraits portent sur l'Afrique (Psichari, Gide, Martin du Gard, Marie Gevers, Louis Charbonneau).

\section{Conclusion}

Que dire de cet aperçu rapide sur l'inscription de Maran dans l'histoire littéraire ? Son nom reste exclusivement associé au prix Goncourt et à Batouala et injustement réduit à une lecture anticolonialiste (la préface y a sans doute largement contribué), idée que retiendront les anthologies qui suivront à l'exception de L. Sainville qui cite des extraits d'autres textes.

Maran est dorénavant rapatrié parmi les auteurs francophones mais sa place, encore une fois, pose question. Les chercheurs en francophonie ont repris et pérennisé la dichotomie français/hors de France, ont maintenu et consolidé l'absence de ressemblance entre certaines aires géographiques où la création en langue française se poursuit, et la France. Seul les rapproche l'idiome qu'elles ont en partage comme si le propre de la littérature ne résidait pas dans la porosité entre les œuvres, un dialogue permanent entre les écrivains.

41 Laissons le dernier mot à l'auteur dont le propos sur son ami Félix Eboué fait étrangement écho à ce qu'il a vécu et subi avec « l'affaire Batouala »: « [e]n fait, que lui reproche-t-on? Tout simplement d'être sorti des sentiers battus, de s'être débarrassé des œillères administratives, d'avoir construit quelque chose de nouveau et de beau, au plus grand honneur de la colonisation française ${ }^{46} »$.

\section{BIBLIOGRAPHIE}

\section{Ouvrages cités}

ALLOUACHE, Ferroudja, Archéologie du texte littéraire dit " francophone » 1921-1970, Paris, Classiques Garnier, 2018

BEAUMARCHAIS, Jean-Pierre, COUTY, Daniel, REY, Alain, Dictionnaire des écrivains de langue française, Paris, Larousse, 1984, 2001

BOURDIEU, Pierre, Les Règles de l'art, Paris, Seuil, coll. « Points », 1992, 1998

CHEVRIER, Jacques, La Littérature nègre, Paris, Armand Colin/VUEF, 1984, 1999 
JARRETY, Michel, La Critique littéraire française au XX ${ }^{e}$ siècle, Paris, PUF, coll. « Que sais-je ? », 1998 JOUBERT, Jean-Louis, Littérature francophone, Paris, Nathan, ACCT/AIF, 1992

JOUBERT, Jean-Louis, Littératures d'Afrique centrale, Paris, Nathan, ACCT/Agence de la francophonie, 1995

KESTELOOT, Lilyan, Histoire de la littérature négro-africaine, Paris, Karthala-AUF, 2001

LAGARDE \& MICHARD, Les Grands auteurs français. XX siècle, Paris, Bordas, 1962, 2001

MARAN, René, Félix Eboué, Grand commis et loyal serviteur, 1885-1944, Paris, Éditions Parisiennes, 1957

MOISAN, Clément, L'Histoire littéraire, Paris, PUF, 1990

Nouvelle Revue française, $\mathrm{n}^{\circ} 1$, janvier 1922

RIESZ, Janos, De la littérature coloniale à la littérature africaine, Paris, Karthala, 2007

SAMOYAULT, Tiphaine, Littérature et mémoire du présent, Nantes, Pleins feux, 2001

SAINVILLE, Léonard, Anthologie de la littérature négro-africaine. Romanciers et conteurs, Paris, Présence africaine, 1963 et 1968

NOUDELMANN, François, Pour en finir avec la généalogie, Paris, Léo Scheer, coll. « Non \& non », 2004

RUBIALES, Lourdes, « Notes sur la réception du Goncourt 1921 en France », Francofonia, n 14, 2005, p. $123-145$

TADIÉ, Jean-Yves (dir.), La littérature française : dynamique et histoire II, Paris, Gallimard, coll. « Folio/ Essais ", 2007

\section{Journaux consultés pour la période d'octobre 1921 à mai 1922}

L'Action française [14-15 déc.]

La Croix [16 déc. $n^{\circ} 11884 ; 18-19$ déc. $\left.n^{\circ} 11886\right]$

L'Écho d'Alger [15 déc. $n^{\circ} 4451 ; 29$ déc. $n^{\circ} 4465 ; 2$ fév. $n^{\circ} 4500 ; 16$ mai n 4603]

L'Écho de Paris [15 déc. $\mathrm{n}^{\circ} 13634$ ]

Le Figaro [31 oct. $\mathrm{n}^{\circ} 304 ; 12$ déc. $\mathrm{n}^{\circ} 342 ; 14-15$ déc. $\mathrm{n}^{\circ}$ 348-349; 23 janv. $\mathrm{n}^{\circ} 23$ ]

Le Gaulois [3 août $\mathrm{n}^{\circ} 16009 ; 16-17$ déc. $\mathrm{n}^{\circ} 16144-16145 ; 17$ janv. $\mathrm{n}^{\circ} 16176$; 19 fév. $\mathrm{n}^{\circ} 16209 ; 22$ fév. $\mathrm{n}^{\circ} 16212 ; 20$ mai $\left.\mathrm{n}^{\circ} 16299\right]$

L’Humanité [6 déc. $\mathrm{n}^{\circ} 6465$; 14-15 déc. $\mathrm{n}^{\circ}$ 6473-6474; 21 fév. $\mathrm{n}^{\circ}$ 6542]

Le Petit Journal [15 déc. $\mathrm{n}^{\circ} 21518 ; 17$ déc. $\mathrm{n}^{\circ} 21520$ ]

Le Petit Parisien [15 déc. $n^{\circ} 16331 ; 23$ déc. $n^{\circ} 16369 ; 27$ déc. $n^{\circ} 16373$ ]

Le Temps [13 déc. $\mathrm{n}^{\circ} 22046$; 15-17 déc. $\mathrm{n}^{\circ} 22048-22050$; 20 déc. $\mathrm{n}^{\circ} 22053$; 18-19 fév.

$n^{\circ} 22112-22113 ; 25$ fév. $\left.n^{\circ} 22119\right]$

\section{NOTES}

1. «BATOUALA, par René Maran (Albin Michel) », rubrique « Le Roman », Nouvelle Revue française, $\mathrm{n}^{\circ} 1$, janvier 1922, p. 103-106.

2. Ibid., p. 106.

3. $\mathrm{N}^{\circ} 11886,18-19$ déc. 1921,4 p. . 
4. «Un éreintement littéraire. Le lauréat du prix Goncourt est sévèrement jugé », $n^{\circ} 4465,29$ déc. 1921, p. 1.

5. $\mathrm{N}^{\circ} 4500,2$ fév. 1922, p. 5.

6. Ibid.

7. Ibid.

8. $\mathrm{N}^{\circ} 4500,2$ février 1922, p. 5.

9. Voir RUBIALES, Lourdes, "Notes sur la réception du Goncourt 1921 en France ", Francofonia, $n^{\circ} 14,2005$, p. 123-145, en ligne sur https://www.redalyc.org/pdf/295/29501409.pdf.

10. $\mathrm{N}^{\circ} 13634$ « Le prix Goncourt à M. René Maran », p. 1 .

11. La Croix, n 11886, 18-19 déc. 1921, p. 4. Nous soulignons.

12. Préface,1 p. 5-19, Paris, Magnard/lycée, 2002.

13. Un exemple pris dans la préface : «Ils n'ont pas voulu voir. Ils n'ont rien voulu entendre. Ils n'ont pas le courage de parler. » Autre extrait du récit: "Je ne me lasserai jamais de dire la méchanceté des blancs. Je leur reproche surtout leur duplicité. [...] », n 6465, 6 déc. 1921.

14. «Entretiens littéraires ", nº 16299, 20 mai 1922, p. 4.

15. Compte rendu de Chaumeix consacré à La Randonnée de Samba Diouf des frères Tharaud (1922).

16. 18-19 déc. $1921, \mathrm{n}^{\circ} 11886$, p. 4.

17. L'Écho de Paris, 15 déc. $1921, \mathrm{n}^{\circ} 13634$, p. 1.

18. L'Écho d'Alger, 2 fév. 1922, n 4500, p. 5.

19. Ibid., 29 déc. $1921, n^{\circ} 4465$, p. 1.

20. Cf. Chap. 5 « Regards critiques sur la société coloniale», RIESZ, Janos, De la littérature coloniale à la littérature africaine, Paris, Karthala, 2007, p. 107.

21. MOISAN, Clément, L'Histoire littéraire, Paris, PUF, 1990, p. 18.

22. L'Écho d'Alger, 2 fév. 1922, n 4500, p. 5.

23. Le Temps, 16 déc. $1921, \mathrm{n}^{\circ} 22049$, p. 1.

24. Le Gaulois, 20 mai 1922, n 16299, Rubrique « Entretiens littéraires », p. 4.

25. Ibid.

26. Paris, Bordas, 1962, rééd. 2001.

27. Quant à l'écrivain suisse Ramuz, il apparaît dans la rubrique "L'homme devant la nature » aux côtés de Bosco et de Colette.

28. P. 579. Les italiques figurent dans le texte.

29. Paris, Gallimard, Folio/Essais, 2007.

30. Ibid., p. 570 et suiv.

31. Cf. p. 603 et suiv.

32. Collection Henri Mitterand, Paris, Nathan, 1998, p. 670-672.

33. Chap. 25, p. 61-686.

34. Faut-il y déceler la rançon de la négritude?

35. Paris, Bordas, 1984, rééd. Larousse 2001. La notice est rédigée par Odile Biyidi, épouse de Mongo Beti (pseudo. de Alexandre Biyidi), p. 1098.

36. Dictionnaire de la littérature française $X X^{e}$ siècle, Paris, Albin Michel, 2000, p. 14.

37. Ibid., la citation est extraite de la préface, p. 5.

38. Ibid., p. 14.

39. Anthologie de la littérature négro-africaine. Romanciers et conteurs, Paris, 1963 et 1968, p. 619.

40. KESTELOOT, Lilyan, Les Écrivains noirs de langue française :: naissance d'une littérature, Bruxelles, Institut de sociologie, Université Libre de Bruxelles, 1963, 1971, p. 83.

41. Le propos trouve un écho dans La Littérature nègre de Chevrier pour qui les figures de proue de la négritude sont Damas (Pigments) et Césaire (Cahier d'un retour au pays natal).

42. Ibid.

43. Ibid.,1 p. 15. 
44. Respectivement parues en 1992 et 1995, Paris, Nathan, ACCT/Agence de la francophonie.

45. P. 194.

46. Félix Eboué, Grand commis et loyal serviteur, 1885-1944, Paris, Éditions parisiennes, 1957, p. 26.

\section{RÉSUMÉS}

La fortune d'un livre et le destin d'un auteur dépendent souvent des commentaires qu'en assurent les chroniqueurs. Bien qu'elle relève d'un jugement de goût, de réactions à chaud, la critique journalistique a une large responsabilité dans la réception d'une œuvre. Notre intervention s'intéresse à la manière dont les critiques bricolent pour évincer Maran et son personnage Batouala de la mémoire de la littérature telle qu'elle est en train de "se faire " à travers la presse de l'époque coloniale (journaux et revues de 1921-1922). Elle interroge aussi la place que lui accordent a posteriori les anthologies littéraires françaises et francophones.

The fate of an author and the book mostly depends on the comments of narrators and archivers. Journalistic criticism is largely responsible for the reception of any works with its judgments which is also including heated reactions. Our intervention primarily aims to oust Maran and his character Batouala from the memory of literature as it is being "made" through the press of the time (newspapers and magazines). It also questions the place accorded to it a posteriori by the French and French literary anthologies.

\section{INDEX}

Mots-clés : mémoire, généalogie, histoire littéraire, intertextualité, francophonie, archives

\section{AUTEUR}

\section{FERROUDJA ALLOUACHE}

FERROUDJA ALLOUACHE est maître de conférences en littératures française et francophones à l'université Paris VIII. Ses domaines de recherche concernent la francophonie et ses rapports avec l'histoire et la mémoire de la littérature, la généalogie, l'archéologie, les archives et presse française durant la période coloniale. 